\title{
FRENECTOMIA LABIAL EM PACIENTE COM SÍNDROME DE CORNELIA DE LANGE
}

\section{LABIAL FRENECTOMY IN PATIENT WITH CORNELIA DE LANGE SYNDROME}

\author{
Amanda Martins de Lima* \\ Caroline Moraes Moriyama** \\ Thami Seles Alves" \\ Maria Teresa Botti Rodrigues dos Santos**** \\ Soraya Carvalho Costa ${ }^{* * * *}$ \\ Renata Oliveira Guaré ${ }^{* * * *}$
}

\section{RESUMO}

Síndrome de Cornelia de Lange é uma anormalidade de desenvolvimento multisistêmica caracterizada por retardo cognitivo e de crescimento; dismorfia craniofacial, malformações dos membros superiores; disfunção geniturinária e oftalmológica, hirsutismo e anomalias cardíacas e gastroesofágicas. Cílios longos e sobrancelhas arqueadas com sinófiris, filtro longo com lábio superior fino. Ponte nasal deprimida com narinas antevertidas também ocorre com frequência. Problemas dentários são frequentes e incluem: palato ogival, micrognatia, atraso na erupção, erosão dental, doença gengival e periodontal. Com base nessas observações, o presente estudo tem como objetivo descrever a frenectomia labial em um paciente com síndrome de Cornelia de Lange, de 19 anos de idade, gênero masculino, bem como as recomendações para o manejo clínico e controle do biofilme dental.

Descritores: Síndrome de Lange • Deficiência intelectual • Doença periodontal

\section{A B S TRACT}

Cornelia de Lange syndrome is an abnormality multisystem developmental characterized by cognitive and growth retardation; craniofacial dysmorphia, malformations of the upper extremities, genitourinary and ophthalmologic dysfunction, hirsutism, cardiac and gastroesophageal abnomalies. Long eyelashes, arched eyebrows with synophrys, long philtrum with thin upper lip. Depressed nasal bridge with anteverted nares also frequently occur. Dental problems are common and include: high palate, micrognathia, delayed eruption, dental erosion, gingival and periodontal disease. Based on these observations, the present study aims to describe the labial frenectomy in a patient with Cornelia de Lange syndrome, 19 years old, male gender, as well as recommendations for the clinical management and control of dental biofilm.

Descriptors: De Lange syndrome • Intellectual disability • Periodontal diseases

\footnotetext{
* Aluna do Doutorado em Odontopediatria da Universidade Cruzeiro do Su

** Aluna do Doutorado em Odontopediatria da Universidade de São Paulo

*** Aluna de Especialização em Saúde da Família UnA-SUS | UNIFESP

**** Disciplina de Pacientes Especiais da Universidade Cruzeiro do Sul
} 


\section{N T R O D U Ç Ã O}

Síndrome Cornelia de Lange (SCL), também conhecida como Brachmann de-Lange síndrome, é uma síndrome de malformação múltipla, congênita de forma autossômica dominante, relativamente rara e de grande diversidade em sua manifestação, afetando entre 1/10.000 e 1/60.000 recém-nascidos, sendo mais frequente em mulheres do que em homens: 1,3/1. Uma grande parte dos casos diagnosticados como SCL parecem ser esporádicos e 10\% dos casos apresentam alterações cromossômicas, a translocação do 3T 26:2 - q23. Em 1916, Brachmann, um pediatra holandês, relatou alguns aspectos dessa síndrome ${ }^{1,2}$, mas foi em 1933, em Amsterdã, que ele descreveu duas meninas com deficiência mental e outras características com uma forma menos grave da mesma síndrome ${ }^{3,4}$.

Classicamente é definida pelo crescimento hipodesenvolvido, deficiência intelectual, retardo do crescimento pré e pós-natal, atraso psicomotor, problemas de comportamento, e malformações do membros superiores ${ }^{5}$. O diagnóstico da SCL é feito clinicamente, já que o indivíduo possui características faciais e físicas distintivas ${ }^{6}$. Os sinais clínicos mais importantes do SCL são: prega palmar simiesca, mobilidade limitada do cotovelo, micromelia, sindactilia. Os olhos podem apresentar ptose palpebral, blefaritis, conjuntivite, estenose do canal palpebral, miopia severa, nistagmo, microcórnea e 30\% sofrem de cardiopatia e hipoacusia, microcefalia, autismo, sinófiris (sombrancelhas arqueadas muito próximas entre si), cílios longos e grossos, orelhas hisurtas com hélices espessas, implantação frontal do cabelo baixo, as orelhas são implantadas baixas, nariz pequeno, narinas antevertidas, filtro labial completo, lábios finos, micrognatia (comissuras inclinadas para baixo) e cianose perioral ${ }^{7,8}$.

Das características bucais, a micrognatia, atresia palatina, microdontia, anadontia parcial, retardo de erupção com espaçamento amplo, hipoplasia de esmalte e estreitamento dos arcos dentais são achados frequentes nesses indivíduos ${ }^{9}$.

Os problemas dentários incluem: pala- to ogival $(30 \%$ apresentam fissuras palatais associadas), pouco desenvolvimento da mandíbula, mau alinhamento dental, erupção dentária retardada, microdontia, doença periodontal e erosão dental pelo refluxo gástrico frequente ${ }^{7,10,11}$. O objetivo do estudo foi relatar um caso de frenectomia labial em indivíduo com síndrome de Cornelia de Lange, de 19 anos de idade, gênero masculino, para facilitar sua higienização oral, influenciando o controle do biofilme dental do paciente.

\section{RELATO DE CASO}

Paciente M.C.T.S., 19 anos, gênero masculino, com diagnóstico de síndrome de Cornelia de Lange. Sua mãe procurou a clínica da Universidade Cruzeiro do Sul em 2006, relatou que não conseguia realizar a higienização bucal adequada, e o mesmo apresentava sangramento gengival. Durante o exame físico geral observou-se baixa estatura, nariz pequeno com narinas antevertidas, deficiência intelectual, sinófiris, cílios longos e lábios finos (Fig.1). Ao exame intrabucal, foram verificados dentes apinhados, gengivite, retração gengival, microstomia, presença de biofilme (Fig. 2) e freio labial pouco evidenciado (Fig. 4), que limitava o movimento do lábio e dificultava a higienização do paciente. Seguiu-se com o plano de tratamento, o qual durou 3 anos, com várias sessões de condicionamento e motivação, incluindo estímulos verbais, visuais, lúdicos, para a sensibilização do paciente da importância da execução da higiene bucal (Fig. 3), procedimentos de raspagem e gengivoplastia. Em 2009 foi realizada a frenectomia de freio labial mediano inferior (Figs. 5 e 6). O tratamento indicado foi eficaz demonstrando estabilidade pós-cirúrgica e após 6 meses, observamos melhora na escovação e ausência de biofilme (Figs. 7 e 8).

\section{I SCUSSÃO}

A síndrome Cornelia de Lange é uma doença genética rara. A maioria dos casos são esporádicos ${ }^{12}$, acometendo tanto homens quanto mulheres independente da raça ${ }^{13}$. O caso que descrevemos apresenta as principais características clínicas
LIMA AM

MORIYAMA CM

ALVES TS

SANTOS MTBR

COSTA SC

GUARÉ RO

FRENECTOMIA

$\angle A B I A L \quad E M$

PACIENTE COM

SINDROME DE

CORNELIA DE

LANGE

171
REV, ODONTOL.

UNIV. CID. SÃo

PAULO

2014; 26(2):

170-6, MAIO-AGO 


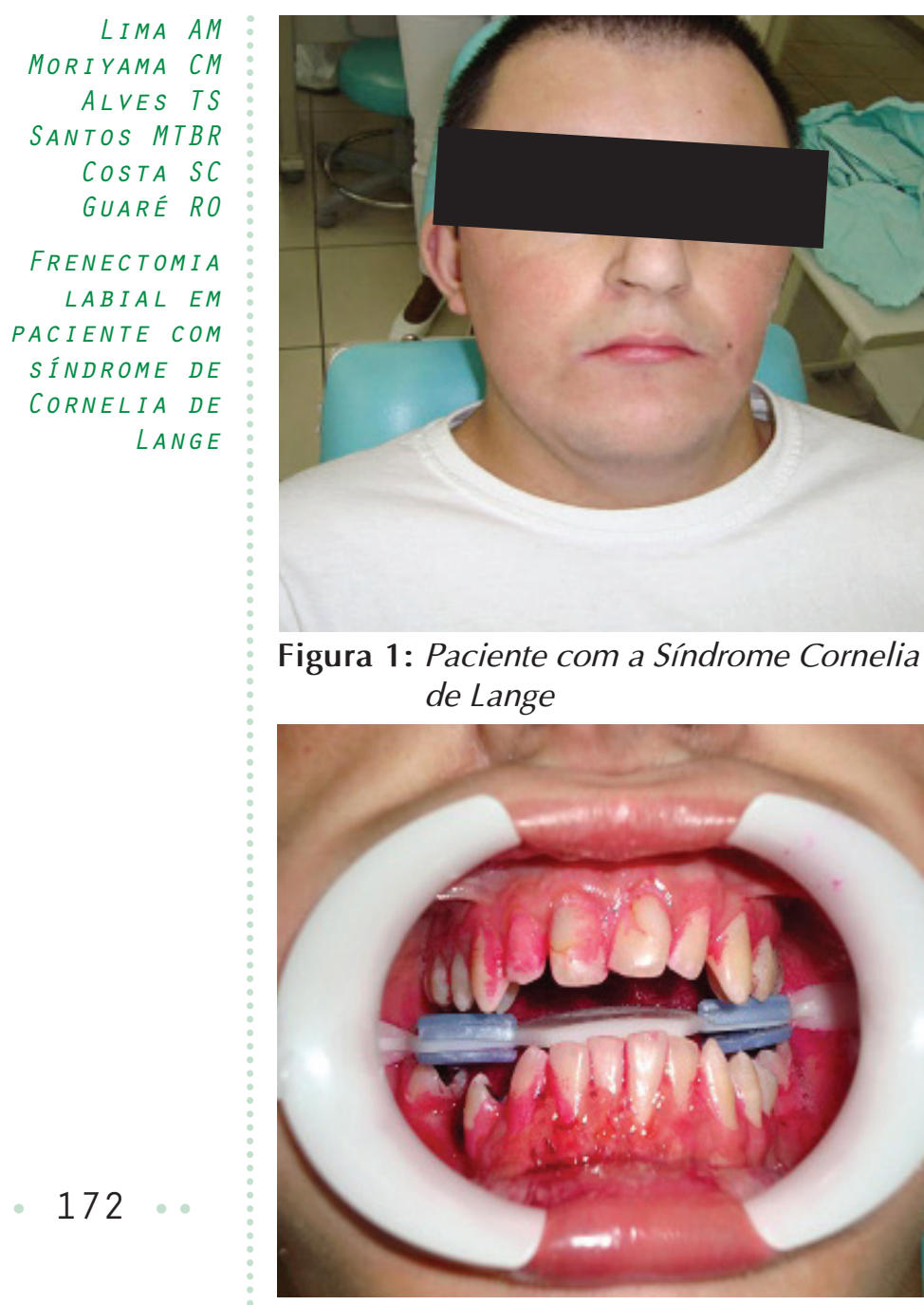

Figura 2: Presença de biofilme

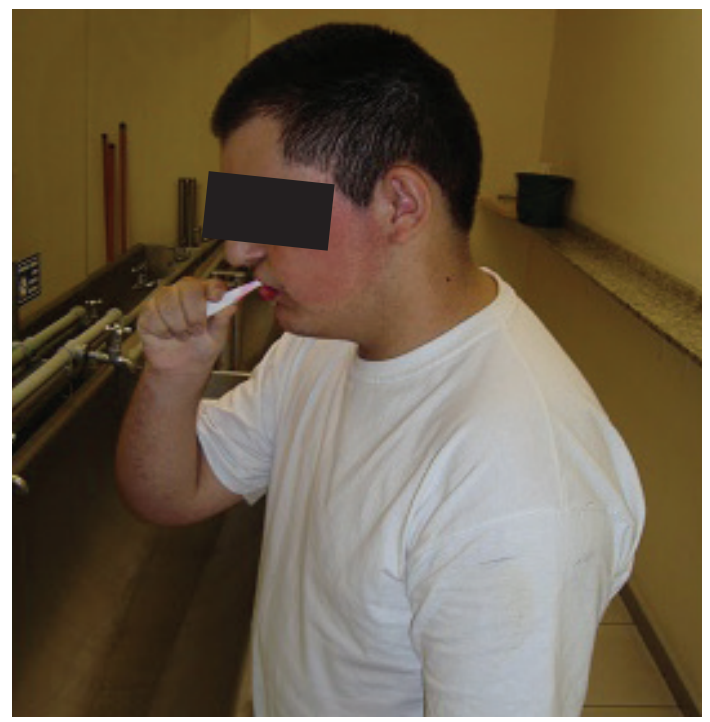

Figura 3: Condicionamento e Motivação

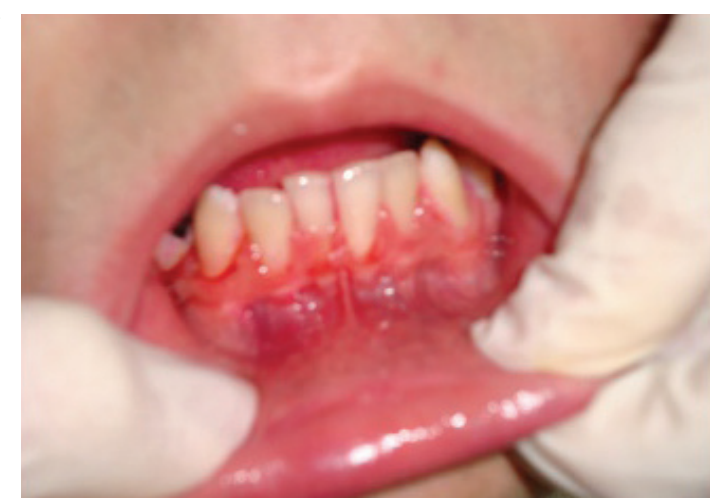

Figura 4: Freio labial curto com inserção baixa na margem gengival

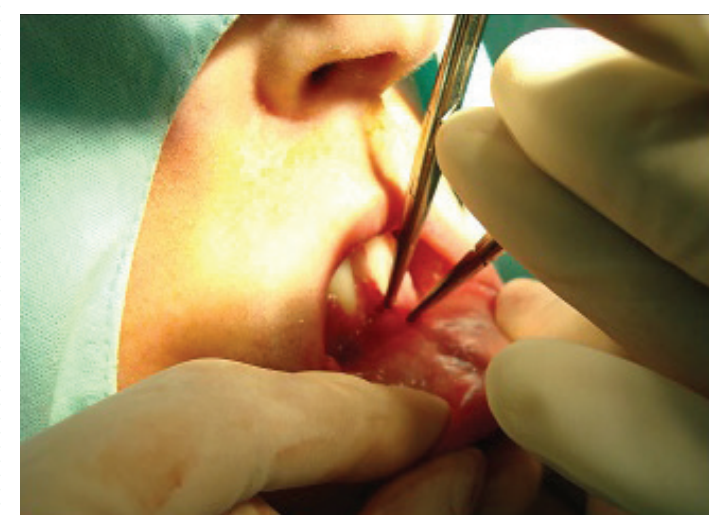

Figura 5: Pinçamento do freio (frenectomia)

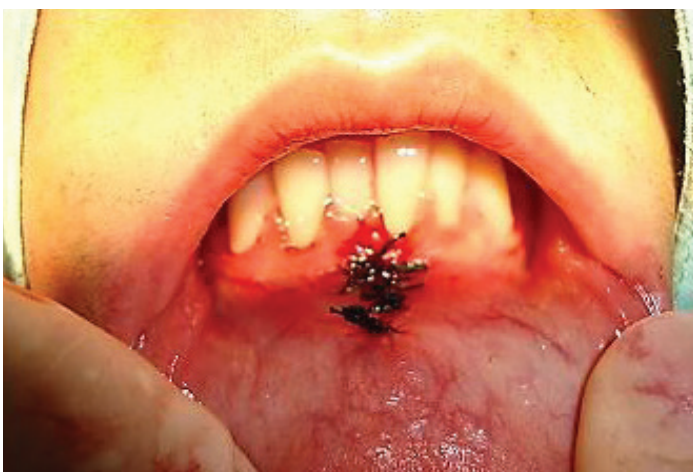

Figura 6: Sutura após frenectomia

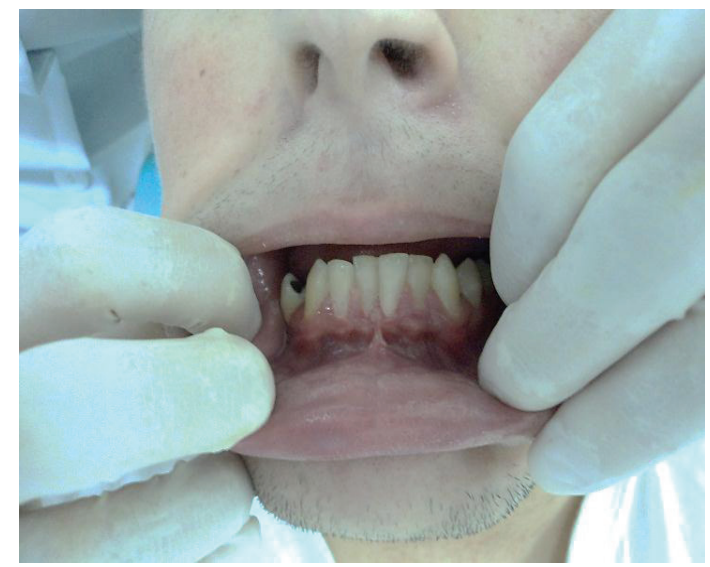

Figura 7: Freio labial evidenciado após a frenectomia
REV, ODONTOL. UNIV, CID. SÃO PAULO $2014 ; 26(2): 170-$ 6. MAIO-AGO 


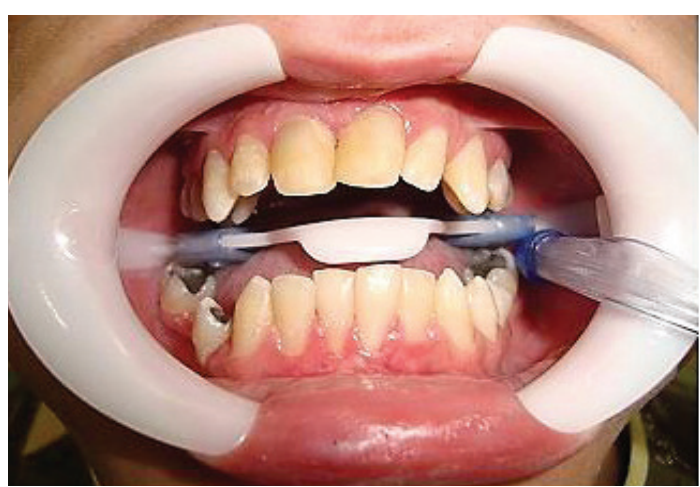

Figura 8: 6 meses após cirurgia, ausência de biofilme

dessa síndrome. A alteração do desenvolvimento e crescimento das maxilas implica a presença de problemas dentários. A deficiência intelectual em conjunto com déficit motor, mau alinhamento dentário, o tipo de dieta e outros fatores fazem com que a presença de problemas periodontais sejam frequentes nesse tipo de paciente. Por essa razão, devem ser observados, desde o início, sangramentos gengivais e deve-se implementar os métodos preventivos ou terapêuticos apropriados.

A higiene oral precária e a má condição bucal registrada estão relacionadas não só aos déficits intelectual e motor, como também ao nível de compreensão da importância da escovação dentária para a saúde bucal, não só do indivíduo como também dos cuidadores ${ }^{14}$, observados no presente estudo.

Uma vez que as doenças bucais mais prevalentes nesses pacientes ${ }^{15}$ são a cárie dentária e a doença periodontal, sendo esta última decorrente de problemas de ordem local, geral, podendo também estar modulada pela utilização rotineira de medicamentos psicoativos, destacando-se os anticonvulsivantes que têm sido menos frequentemente associados aos crescimentos gengivais, como é o caso do nosso paciente que, apesar de nunca ter convulsionado, utilizava Ácido Valproico $250 \mathrm{mg}^{16}$.

A colaboração do paciente é indispensável, considerando-se as manipulações por via oral, para determinar as técnicas complementares para manusear o comportamento ideal, em cada caso ${ }^{17}$. Houve colaboração do nosso paciente e da sua cuidadora, por esse motivo foi realizado o tratamento periodontal, levando-se em conta a preocupação da mãe em cuidar da saúde bucal do seu filho. Revisões de rotina mensais facilitaram as mudanças no periodonto e o cuidado com os dentes em casa.

A inserção na gengiva marginal ou próxima a ela é apontada como causadora de retração gengival, principalmente no arco inferior; no entanto, o envolvimento do frênulo, em muitos casos, ocorre tardiamente, além de outros fatores predisponentes encontrados com frequência nessa área, como posição proeminente dos dentes no $\operatorname{arco}^{18-20}$ e ausência de gengiva inserida ${ }^{18}$, além de vestíbulo raso, que dificulta a higiene ${ }^{19}$.

Diversos autores ${ }^{21-23}$ apontam que a existência de um frênulo que tracione a gengiva marginal pode causar muitos transtornos; no entanto, Powell e Mceniery $^{19}$ (1982) afirmam que a posição do frênulo pode meramente acelerar o processo ou, como afirma Bress$\operatorname{man}^{23}$ (1973), «O frênulo pode agravar ou potencializar a desorganização periodontal adicional quando a doença periodontal está presente».

A influência do frênulo/freio depende muito da arcada dentária, sendo que a posição do frênulo labial superior é relativamente sem importância para o acúmulo de placa e sangramento. Alguns estudos sugerem que a inserção do frênulo labial inferior que invade a gengiva marginal afeta a retenção da placa e a prevalência da gengivite $^{18}$. Dessa forma, em termos clínicos para placa e sangramento, com a dificuldade motora para a correta higienização oral do paciente e da sua cuidadora, o freio curto garantia piora na doença periodontal.

Leite Neto et al. ${ }^{24}$ (1975) não encontraram correlação estatisticamente significante entre a altura do frênulo labial inferior e a eficiência da higiene bucal, o que corrobora os achados deste caso. Contudo, pode ser questionado o fato de que o autor não realizou pesquisas em SCL. Porém, há também controversas, quando observamos nas imagens que o paciente apresentava sangramento gengival em toda a extensão da arcada e não somente na região de incisivos centrais inferiores.
LIMA AM

MORIYAMA CM

ALVES TS

SANTOS MTBR

COSTA SC

GUARÉ RO

FRENECTOMIA $\angle A B I A L \quad E M$

PACIENTE COM

SÍNDROME DE

CORNELIA DE

LANGE

\section{3}

REV, ODONTOL.

UNIV. CID. SÃO PAULO

2014; 26(2):

170-6, MAIO-AGO 
LIMA AM : MORIYAMA CM ALVES TS

SANTOS MTBR

COSTA SC

GUARE RO

FRENECTOMIA

$\angle A B I A L \quad E M$

PACIENTE COM

SINNDROME DE

CORNELIA DE

LANGE

$174 \ldots$

REV, ODONTOL.

UNIV, CID, SÃO PAULO

$2014 ; 26(2): 170-$

6. MAIO-AGO
Neste caso relatado, foram realizadas motivações supervisionadas. Assim, pôde-se verificar que, com a frenectomia e a mudança da posição de inserção do frênulo, foi possível se obter o controle adequado do biofilme, conseguindo-se, então, um índice de gengivite compatível com normalidade clínica. Esse aspecto encontra um diferente respaldo em uma afirmativa de Ramfjord et al. ${ }^{25}$ (1991), os quais opinam que "o que se deve levar em consideração não é a posição do frênulo e, sim, a possibilidade de o indivíduo manter boa condição de higiene bucal".

Para o cirurgião-dentista, o planejamento clínico é de extrema importância, pois em casos de $\mathrm{SCL}$, o profissional deve estar atento às limitações físicas e mentais do paciente e, assim, proporcionar uma melhora na qualidade de vida deste, iniciando pela promoção e prevenção de sua saúde bucal. Neste relato de caso, observamos as características fenotípicas mencionadas por Ireland et al. ${ }^{26}$ (1993) e também aspectos autísticos relatados por Barr et al. ${ }^{27}$ (1971). A deficiência mental, em conjunto com déficit motor, apinhamento dental, o tipo de dieta e outros fatores, fazem com que a presença de problemas periodontais sejam frequentes nesse tipo de paciente.

Santos, Fernandes e Biziak ${ }^{28}$ (2001) mencionaram em seu estudo um paciente SCL que foi submetido a procedimento cirúrgico de ulectomia sob anestesia local, permitindo, dessa forma, a erupção dos incisivos superiores e aumento da amplitude da cavidade oral, facilitando a deposição dos alimentos na lateral, tornando a mastigação mais eficiente. Entretanto, Carbó et al. ${ }^{14}$ (2007) relataram que só foram possíveis as exodontias e, posteriormente, o tratamento periodontal do paciente sob anestesia geral, pois não havia cooperação deste. No caso relatado, o paciente permitiu a interação dos profissionais na escovação, bem como na cirurgia da frenectomia após o condicionamento e motivação adequados.

\section{CONCLUSÃO}

Analisando o relato clínico e a revisão literária deste estudo, pode-se concluir que:

- A frenectomia labial inferior no paciente com Síndrome Cornelia de Lange foi comprovadamente necessária.

- A higiene oral nesses indivíduos com défict psicomotor é realmente precária, favorecendo acúmulo de biofilme, presença de cárie e doença periodontal.

- A preocupação e a colaboração do cuidador, juntamente com o planejamento correto da equipe odontológica, são extremamente importantes para o sucesso do tratamento.

- Foi possível, no período de tratamento, manter um controle do biofilme dental compatível com a normalidade clínica gengival. 
1. O'Donnell D, Davis PJ, King NM. Management problems associated with Cornelia de Lange syndrome. Spec Care Dentist 1985 Jul-Aug;5(4):160-3.

2. Brachmann W. Ein fall von symmetrischer monodaktylie durch Ulnadefekt, mit symmetrischer flughautbildung in den ellenbeugen, sowie anderen abnormitaten (zwerghaftogkeit, halsrippen, behaarung). Jarb Kinder Phys Erzie 1916 84):225+35.

3. Gilgenkrantz S. [Cornelia de Lange syndrome]. Med Sci (Paris) 2004 Nov;20(11):954-6.

4. De Lange C. Sur un type nouveau de degenerescence (typus Amstelodamensis). Arch Med Enfants 1933 36(713-9.

5. Cruz M, Bosch J. Atlas de síndromes pediátricos. Barcelona: Expaxs; 1998.

6. Kline AD, Krantz ID, Sommer A, Kliewer M, Jackson LG, FitzPatrick DR, et al. Cornelia de Lange syndrome: clinical review, diagnostic and scoring systems, and anticipatory guidance. Am J Med Genet A 2007 Jun 15;143A(12):1287-96.

7. Smith D. Atlas de malformaciones somáticas en el niño. Barcelona: Editorial Pediátrica; 1978.

8. Raspall G. Enfermedades maxilares y craneofaciales: atlas clínico. Barcelona: Salvat Editores; 1990.

9. Yamamoto K, Horiuchi K, Uemura K, Shohara E, Okada Y, Sugimura M, et al. Cornelia de Lange syndrome with cleft palate. Int J Oral Maxillofac Surg 1987 Aug;16(4):484-91.

10. Cebrian-Carretero JL, Lopez-Arcas-Calleja JM. Gastroesophageal reflux diagnosed by occlusal splint tintion. Med Oral Patol Oral Cir Bucal 2006 Jan;11(1):E26-8.

11. Sataloff R, Spiegel J, Hawkshaw M, Epstein JM, Jackson L. Cornelia de Lange syndrome. otolaryngologic manifestations. Arch Otolaryngol Head Neck Surg 1990 Sep.;116(9):1044-6.
12. Borck G, Redon R, Sanlaville D, Rio M, Prieur M, Lyonnet S, et al. NIPBL mutations and genetic heterogeneity in Cornelia de Lange syndrome. J Med Genet 2004 Dec;41(12):e128.

13. McConnell V, Brown T, Morrison PJ. An Irish three-generation family of Cornelia de Lange syndrome displaying autosomal dominant inheritance. Clin Dysmorphol 2003 Oct;12(4):2414.

14. Grau Carbo J, Lopez Jimenez J, Gimenez Prats MJ, Sanchez Molins M. Cornelia de Lange syndrome: a case report. Med Oral Patol Oral Cir Bucal 2007 Oct;12(6):E445-8.

15. Machado M. Etiologia dos aumentos gengivais. Rev de Periodontia. 2001;11(3):23-9. Rev de Periodontia 2001 jun.;11(3):23-9.

16. Moschini V, Ambrosini MT, Sofi G. [Anesthesiologic considerations in Cornelia de Lange syndrome]. Minerva Anestesiol 2000 Nov;66(11):799806.

17. Corn H. Technique for repositioning the frenum in periodontal problems. Dent Clin North Am, 1964 mAR.;1(3):79.

18. Addy M, Dummer PM, Hunter ML, Kingdon A, Shaw WC. A study of the association of fraenal attachment, lip coverage, and vestibular depth with plaque and gingivitis. J Periodontol 1987 Nov; 58(11):752-7.

19. Powell RN, McEniery TM. A longitudinal study of isolated gingival recession in the mandibular central incisor region of children aged 6-8 years. J Clin Periodontol 1982 Sep;9(5):357-64.

20. Baer P, Benjamin S. Enfermedad periodontal en niños y adolescentes. Buenos Aires: Mundi; 1975.

21. Carranza F. Periodontia clínica de Glickman. 5 ed. Rio de Janeiro: Guanabara Koogan; 1986.
LIMA AM

MORIYAMA CM

ALVES TS

SANTOS MTBR

costa sC

GUARÉ RO

FRENECTOMIA

LABIAL EM

PACIENTE COM

SÍNDROME DE

CORNELIA DE

LANGE

175
REV, ODONTOL.

UNIV. CID. SÃo

PAULO

2014; 26 (2):

$170-6$, MAIO-AGO 
LIMA AM MORIYAMA CM

$A L V E S T S$

SANTOS MTBR

COSTA SC

GUARE RO

FRENECTOMIA

$\angle A B I A L \quad E M$ PACIENTE COM SINDROME DE CORNELIA DE $\angle A N G E$
22. Goldman H, Cohen D. Periodontia. Rio de Janeiro: Guanabara Koogan; 1983.

23. Ward H. A periodontal point of view. Springfield: Charles C. Thomas; 1973.

24. Leite N. O índice de placa bacteriana relacionado à altura do frênulo labial inferior. Rev Fac Odontol Univ São Paulo 1975 jul.-dez.;13(2):149-54.

25. Ramfjord S, Ash M. Periodontologia e Periodontia. São Paulo: Santos; 1991.

26. Ireland M, Donnai D, Burn J. Brachmann-de Lange syndrome. Delineation of the clinical phenotype. $A m$ J Med Genet 1993 Nov 15;47(7):95964.
27. Barr AN, Grabow JD, Matthews CG, Grosse FR, Motl ML, Opitz JM. Neurologic and psychometric findings in the Brachmann-De Lange syndrome. Neuropadiatrie 1971 Jul;3(1):46-66.

28. Santos MTBR, Fernandes AS, Biziak TR. Síndrome Cornélia de Lange: enfoque interdisciplinar. J Bras Odontopediatr Odontol Bebe 2001 setout.;4(21):419-23.

Recebido:10/08/2014

Aceito: 11/03/2015
REV. ODONTOL. UNIV, CID. SÃO PAULO 2014; 26(2): 170 6, MAIO-AGO 